\title{
Analysis of Stiffness and Damping Properties of Active Magnetic Bearing Using Cross Feedback Control
}

\author{
Peng-Cheng PU, Jin-Peng YU and Lei ZHAO \\ Institute of Nuclear and New Energy Technology \\ Collaborative Innovation Centre of Advanced Nuclear Energy Technology \\ Key Laboratory of Advanced Reactor Engineering and Safety of Ministry of Education \\ Tsinghua University, Beijing 100084, China
}

Keywords: Active magnetic bearing, Cross feedback control, Stiffness, Damping.

\begin{abstract}
Stiffness and damping properties of the magnetic bearings are mainly determined bythe parameters of system controller, both of which are the function of rotor frequency. In the system of magneticsuspensionflywheel (MSFW), the rotor applied has a large moment of inertia ratio, resulting in a strong gyroscopic effect at high speed. Decentralized PID controller cannot assure the stability of suspension at high speed. Therefore, cross feedback control must be added to the control loop of the system. The cross stiffness and damping terms will be introduced to the matrix of stiffness and damping of the system. In this paper, the magnetic bearing rotor system with displacement and velocity cross feedback is discussed from the perspective of the stiffness and damping. The results show that, equivalent stiffness of the rotor approximates a negative constant in low frequency, and with rotation increasing, it increases continually after approaching to zero, Equivalent rotor damping approximates a constant, and decreases continually in high frequency with rotation speed increasing. The filters have influence on stiffness and damping characteristics of magnetic bearing in different frequency range.
\end{abstract}

\section{Introduction}

AMB (active magnetic bearing) is a new type of bearing with high performance. The rotor is levitated by controllable electromagnetic force with no contact. AMB enables the rotor to rotate in super high speed, and has been widely used in high speed rotating machinery, owing to its characteristics of no friction, no lubrication, high precision, long life and active controllable damp and stiffness[1].

In the magnetic suspending flywheel system, the precession frequency of the rotor continuously reduces with the increasing rotational speed. It is very difficult to damp the precession of the rotor. The time delay in the system reduces the stability, even causes instability. In addition, the nutation frequency of the rotor increases with increasing rotational speed. For the flat rotor like flywheel, nutation frequency will be much higher than the synchronized frequency of the rotor. It is very difficult to increase the damping of precession and nutation at high speed by merely adjusting the PD controller parameters [2].

In the high-speed magnetic suspending flywheel system, the rotor applied has a large moment of inertia ratio, resulting in strong gyroscopic effect at high speed. Decentralized PID controller cannot assure the stable suspension for high-speed magnetic suspending flywheel, which requires phase compensation for whirling motion at high speed. For precession motion, reducing the integral coefficient and increasing the differential coefficients can improve the situation of phase delay in low frequency. However, the precession mode will still approach the critical stability with the speed increasing. Similarly, as for nutation motion, increasing the differential coefficient, the system bandwidth and the phase lead can improve the phase delay situation in high frequency. However, in practical system, these methods may introduce high frequency noise. As a result, excessively high frequency gain may lead to the power amplifier saturation. Meanwhile, other factors in the system, like the delay procession, limit the application of these methods. With relatively simple structure, cross feedback control provides compensation on the precession and nutation instability generated by gyroscopic effects, which is based on decentralized control added with cross feedback paths. 
Electromagnetic bearing is a highly complicated coupling system of mechanism, electricity and magnetism, and its bearing stiffness and damping are important parametersof the system performance. Therefore, it is significant to select appropriate parameters for getting high performance. Meanwhile, additional burden for the system also can be reduced. Haberman, et al [3] found that magnetic bearings have stiffness curve similar to "bath box" in 1980. Hereafter, most scholars analyse the stiffness characteristics of magnetic bearing with the found of Haberman. Humphris, et al [4] derived equivalent stiffness and equivalent damping coefficient basing on the transfer function of electromagnetic bearing system. With the analysis theory and method based on the stiffness and damping characteristic of system transfer function Yu Liang, et al [5] established the theory and method for analysing the rotor dynamic characteristic of electromagnetic bearing, and validated it by developing and testing a practical oxygen turbine expander prototype. Zhao Jingjing, et al [6] developed a detecting system to get the stiffness and damping of electromagnetic bearing easily. The system can have the stiffness and damping detected automatically. Moreover, the design process can be simplified by choosing controller parameters with the conception of equivalent stiffness and damping. Bauomy [7] investigated the response of the AMB-rotor system subjecting to a periodically time-varying stiffness. The stability of system near the simultaneous combined and sub-harmonic resonance was analysed by the 4th Rung-Kutta method. Sayyad [8] presented the use of a variable stiffness type magnetic vibration absorber to control the vibration of a beam structure. Its principle is similar to the rotor vibration adjusting of AMB with variable stiffness in single DOF. Shiqiang Zheng [9] presented an optimization of damping compensation for a flexible rotor to make maximal use of the limited electromagnetic force to retain flexible deformation. The optimal compensation phase angle with and without considering the gyroscopic effect is derived. Yuanping $\mathrm{Xu}$ et al [10] proposed a rotor unbalance response based approach to identifying the AMBs stiffness and damping coefficients during rotation. The AMBs dynamic force parameters (stiffness and damping) are verified by comparing the estimated and experimental rotor unbalance responses, which shows high accuracy.

Current researches on equivalent stiffness and damping of magnetic bearing mainly focus on single degree of freedom, which is not suitable for the rotor with high gyroscopic effect. Therefore, the system must be overall analysed with consideration of the influence on stiffness and damping characteristic caused by cross feedback control.

In this paper, the active magnetic bearing with four degrees of freedom (DOF) in radial direction is studied. The controller of the system consists of a decentralized PID controller with cross feedback control paths. Taking intoconsideration the influence of cross feedback control on bearing characteristics of the rotor, this paper analyses how equivalent stiffness, equivalent damping and dynamic stiffness of the rotor changein the different frequencyrange.

\section{Model of Magnetic Suspension Flywheel}

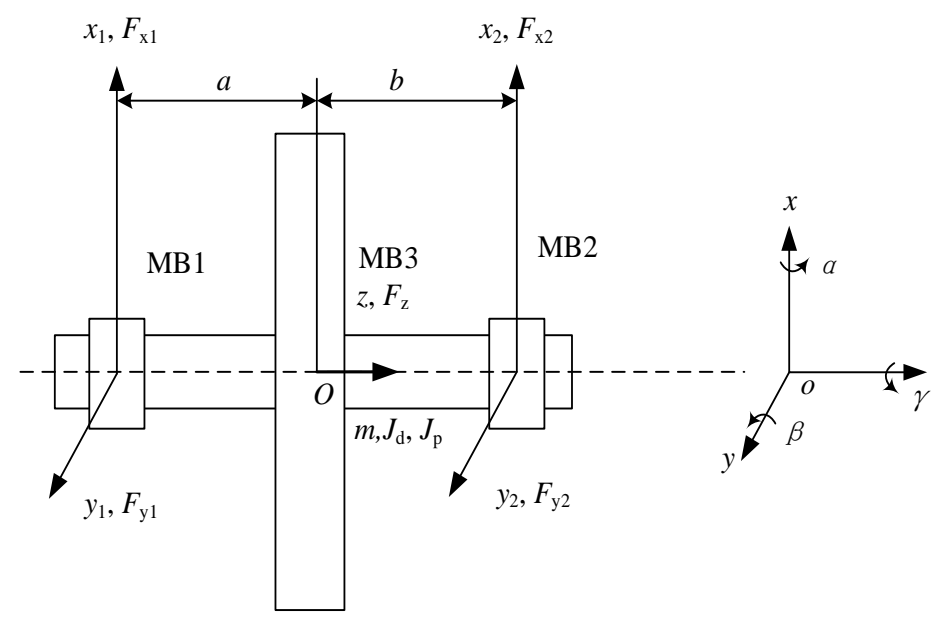

Figure 1.Force analysis model of flywheel rotor. 
Force analysis of the simplified magnetic suspending flywheel is shown in figure1. Radial magnetic bearing MB1, MB2 and MB3 apply electromagnetic force on the rotor. Considering the decoupling radial and axial directions, only radial forces are studied in this paper. Define the mass centre coordinate as $q=[\beta, x,-\alpha, y]^{T}$, bearing coordinate $q_{b}=\left[x_{1}, x_{2}, y_{1}, y_{2}\right]^{T}$ and bearing force $u_{f}=\left[F_{x_{1}}, F_{x_{2}}, F_{y_{1}}, F_{y_{2}}\right]^{T}$.

The dynamical equation of model in the figure 1 can be obtained as:

$$
M \ddot{q}+G \dot{q}=B u_{f}
$$

In equation (1), $M$ is the generalized mass matrix, $G$ the gyroscope matrix, ${ }^{q}$ the mass centre coordinate vector and ${ }^{u_{f}}$ the bearing force. The specific expression is as follows:

$$
M=\left[\begin{array}{cccc}
J_{d} & 0 & 0 & 0 \\
0 & m & 0 & 0 \\
0 & 0 & J_{d} & 0 \\
0 & 0 & 0 & m
\end{array}\right], G=\left[\begin{array}{cccc}
0 & 0 & J_{p} \Omega & 0 \\
0 & 0 & 0 & 0 \\
-J_{p} \Omega & 0 & 0 & 0 \\
0 & 0 & 0 & 0
\end{array}\right],
$$

The bearing force after linearization can be written as:

$$
u_{f}=-K_{s} q_{b}+K_{i} I_{i}
$$

In Equation (2), $K_{s}=\operatorname{diag}\left(k_{s}, k_{s}, k_{s}, k_{s}\right)$ is the matrix of force/displacement, $K_{i}=\operatorname{diag}\left(k_{i}, k_{i}, k_{i}, k_{i}\right)$ the matrix of force/current and $I_{i}=\left[\begin{array}{llll}i_{x 1} & i_{x 2} & i_{y 1} & i_{y 2}\end{array}\right]^{T}$ the Control current.

Define $G_{r}(\mathrm{~s})$ as controller of electromagnetic bearing. The structure of controller contains cross feedback and not all off-diagonal elements are zero. The control current of magnetic bearing is:

$$
I_{i}(s)=G_{r}(s) q_{b}(s)
$$

Then:

$$
\begin{gathered}
M \ddot{q}+G \dot{q}=B\left(K_{i} I_{i}+K_{s} q_{b}\right) \\
M s^{2} q(s)+G s q(s)=B\left[K_{i} G_{r}(s)+K_{s}\right] q_{b}(s)
\end{gathered}
$$

Define equivalent stiffness and damping matrix of the rotor as $K \in R^{4 \times 4}, D \in R^{4 \times 4}$ :

$$
M \ddot{q}+G \dot{q}=B u_{f}=-B\left(K q_{b}+D \dot{q}_{b}\right)
$$

Hence for ${ }^{s}=j \omega$, the stiffness matrix $K(\omega)$ and damping matrix $D(\omega)$ can be obtained:

$$
\left\{\begin{array}{l}
K(\omega)=-K_{i} \operatorname{Re}\left[G_{r}(j \omega)\right]-K_{s} \\
D(\omega)=-\frac{K_{i} \operatorname{Im}\left[G_{r}(j \omega)\right]}{\omega}
\end{array}\right.
$$

In Equation (7), $\operatorname{Re}\left[G_{r}(j \omega)\right]$ represents real component of the $G_{r}(j \omega), \operatorname{Im}\left[G_{r}(j \omega)\right]$ the imaginary component of the $G_{r}(j \omega), \omega$ the vibration frequency of rotor.

The dynamic characteristic of the supported rotor under each degree of freedom is:

$$
\frac{F_{d}(\omega)}{x(\omega)}=K_{d y}=\sqrt{\left(k-m \omega^{2}\right)^{2}+(d \omega)^{2}}
$$

In equation $(8), F_{d}(\omega)$ is the external disturbance force applied on the rotor, $K_{d y}$ the dynamic stiffness of bearing- rotor system. 
According to Equation (8) and (9), it can be inferred that the stiffness, damping and dynamic characteristics of the magnetic bearing system are mainly determined by the frequency characteristics of the controller parameters in addition to the structural parameters.

Basing on the above analysis, the expression for stiffness and damping matrix of bearing system can be obtained by adding ideal velocity and displacement cross feedback to PID control. Cross feedback only has influence on off-diagonal elements of stiffness matrix and damping matrix. In practical system, the ideal cross feedback is hard to achieve. The system is lack of stability margin and dynamic characteristic of the rotor is unsatisfactory. Therefore, the control structures need to be optimized reasonably.

\section{The Principle of Cross Feedback Control}

Decentralized PID controller cannot assure stability suspension of the high-speed magnetic suspending flywheel. Cross feedback control will solve this problem effectively. Displacement and velocity cross feedback control are two available ways. Figure 2 shows a simple realization of a decentralized controller augmented by an additional feedback path for compensation of the gyroscopic cross coupling. The computation time for the system will not increase much since the controller expansion is small. Furthermore, control parameters can be found based on physical interpretation.

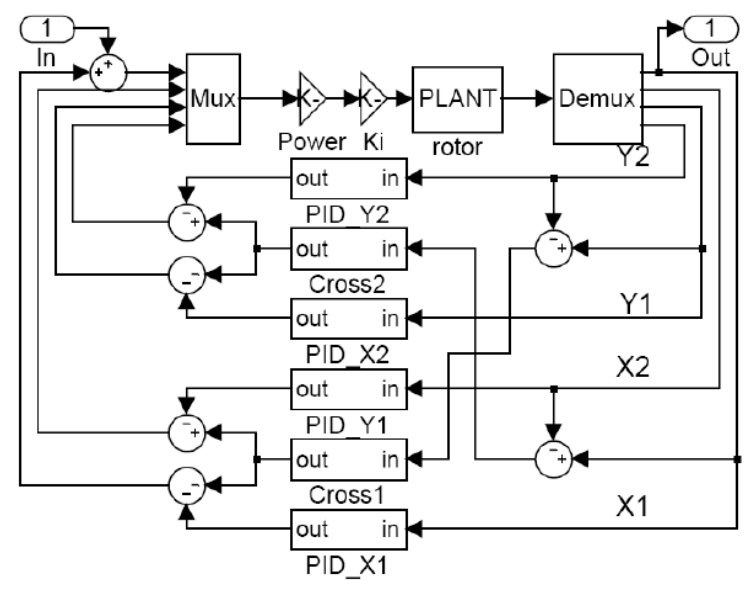

Figure 2.The controller structure with cross feedback.

\section{Displacement Cross Feedback Control}

Displacement cross feedback generates the control variable by introducing displacement to the rotor precession and nutation in orthogonal directions. And the feedback has damping effect on conical motion of the rotor, which improves the stability of rotor nutation and precession. Define ${ }^{k_{c}}$ as the displacement cross coefficient, and it can be positive or negative. After introducing cross feedback control paths, the characteristic equation of rotor nutation and precession can be obtained as follows:

$$
\left\{\begin{array}{l}
J_{d} \ddot{\alpha}+J_{p} \Omega \dot{\beta}+0.5 k l^{2} \alpha+k_{c} \beta=0 \\
J_{d} \ddot{\beta}-J_{p} \Omega \dot{\alpha}+0.5 k l^{2} \beta-k_{c} \alpha=0
\end{array}\right.
$$

Defining $\varphi=\alpha+i \beta$, the characteristic equation can be obtained:

$$
J_{d} s^{2}-J_{p} \Omega i s+0.5 k l^{2}-k_{c} i=0
$$

The roots of the characteristic equation can be shown as follows:

$$
s=\frac{J_{p} \Omega i \pm i \sqrt{J_{p}{ }^{2} \Omega^{2}+4 J_{d}\left(0.5 k l^{2}-k_{c} i\right)}}{2 J_{d}}
$$




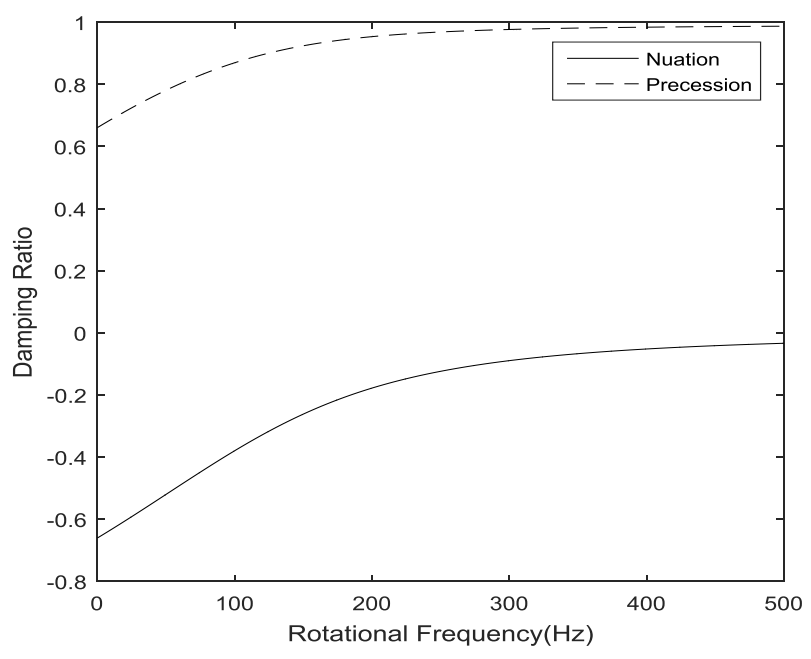

Figure 3.The damping ratio of positive displacement cross feedback as a function of rotation speed.

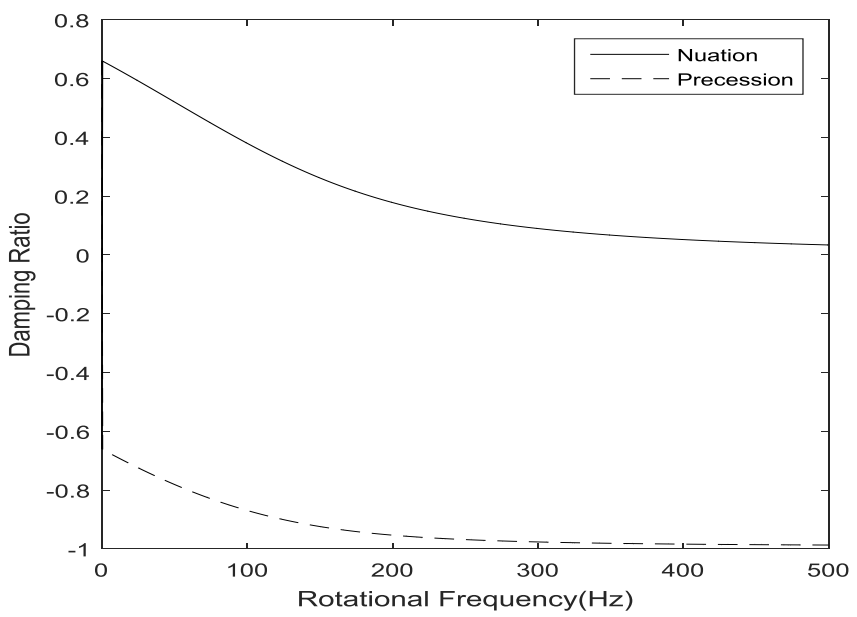

Figure 4.The damping ratio of negative displacement cross feedback as a function of rotation speed.

If the displacement feedback coefficient is not too large, it has little impact on the modal frequency of rotor precession and nutation, while significantly influencingthe value of damping. Positive displacement cross coefficient has a good damping effect on the precession of the rotor, and the effect enhances with rotational speed increasing. However, positive coefficient causes the negative damping on nutation, which means it will motivate the nutation. On the contrary, negative displacement crosscoefficient a has good damping effect on the nutation of the rotor, and the effect weakens with rotational speed increasing. Meanwhile, negative coefficient causes the negative damping on precession, which means it may motivate the precession.

In order to eliminate the nutation and precession simultaneously, positive and negative displacement cross feedback parameters can be introduced at the same time. With the increase of rotor speed, the precession is in the low frequency range while the nutation in the high frequency range. Therefore, the positive displacement cross feedback coefficient can be designed to work only in the low frequency range, while the negative one works only in the high range. Two types of cross feedback control can simultaneously eliminate nutation and precession well with high-pass and low-pass filter.

In the practical control system Transfer function of displacement cross feedback paths can be obtained as:

$$
G(\mathrm{~s})=\frac{k_{c l}}{T_{l p} s+1}+\frac{k_{c h} s}{T_{h p} s+1}
$$


In Equation (12), LP cross feedback coefficient is $k_{c l}$, cut-off frequency of LP cross feedback is $T_{l p}$, HP cross feedback coefficient is $k_{c h}$ and cut-off frequency of LP cross feedback $T_{h p}$.

\section{Velocity Cross Feedback Control}

Velocity cross feedback generates the feedback force for one DOF with the velocity signal in the orthogonal direction. With velocity cross feedback added, the rotor dynamic function can be written as:

$$
\left\{\begin{array}{l}
J_{d} \ddot{\alpha}+\left(1-k_{v}\right) J_{p} \Omega \dot{\beta}+0.5 k l^{2} \alpha=0 \\
J_{d} \ddot{\beta}-\left(1-k_{v}\right) J_{p} \Omega \dot{\alpha}+0.5 k l^{2} \beta=0
\end{array}\right.
$$

Define $^{k_{v}}$ as velocity cross feedback coefficient, which means the compensation for the gyroscopic effect.

Defining $\varphi=\alpha+i \beta$, the characteristic equation can be obtained as:

$$
J_{d} s^{2}-\left(1-k_{v}\right) J_{p} \Omega i s+0.5 k l^{2}=0
$$

The roots of the characteristic equation canbe shown as follows:

$$
s=\frac{\left(1-k_{v}\right) J_{p} \Omega i \pm \sqrt{-\left[\left(1-k_{v}\right) J_{p} \Omega\right]^{2}-2 J_{d} k l^{2}}}{2 J_{d}}
$$

The gyroscopic moment of the rotor can be compensated by velocity cross feedback to avoid instability, with the increase of the precession frequency and decline of the Nutation frequency. If $k_{v}=1$, the gyroscopic effect can be compensated completely.

For magnetic suspending flywheel, nutation has high frequency while precession has low frequency, which is the main difficulty to restrain them simultaneously in high speed rotor. In essence, the difficulty is raised from gyroscopic torque. Therefore, if the gyroscopic effect can be weakened to decrease nutation frequency and increase precession frequency, the system stability can be easily achieved. Velocity cross feedback control is based on the method stated. To reduce the gyroscopic effect by increasing the moment in opposite direction, the control variables are generated with the speed signal introduced in orthogonal direction, which will decrease the nutation frequency and increase the precession frequency.

In theory, if the gyroscopic torque can be restrained completely, the nutation and precession frequency will not change with the speed. However, no ideal speed observer exists in the practice system. The speed observations in use always possess phase lag, and the problem become worse in the high frequency range, resulting in the difficulty to get desired speed signal directly. Furthermore, the time delay module in the system will affect the velocity cross feedback.

First-order differentiator $D s /(T s+1)$ is applied as the speed observer in practical. The phase approaches to zero in high frequency. The observer structure is similar to the negative displacement cross feedback with high-pass filter.

\section{Simulation Results on Stiffness and Damping Properties of Cross Feedback Control}

After the previous analysis, it can be seen that the transfer function of the practical cross feedback path:

$$
G(\mathrm{~s})=\frac{k_{c l}}{T_{l p} s+1}+\frac{k_{c h} s}{T_{h p} s+1}
$$

The relative parameters adopted in the simulation belong to a high-speed magnetic suspension flywheel: 
$m=9.3 \mathrm{~kg} ; k_{c l}=800 ; T_{l p}=1 /(2 \times \pi \times 10) ; k_{c h}=-8 ; T_{h p}=1 /(2 \times \pi \times 300) ; k_{i}=53.6 \mathrm{~N} / \mathrm{A} ; k_{s}=1.8 \times 10^{5} \mathrm{~N} / \mathrm{m}$

\section{Positive Low-Pass Displacement Cross Feedback Path}

For the procession of the rotor, positive displacement cross feed back with low-pass characteristics, namely $G_{l}=\frac{k_{c l}}{T_{l p} s+1}$, has low gain in medium and high frequency range, which can damp the precession effectively while the damping of the nutation is small.

$$
G(j \omega)=\frac{k_{c l}}{T_{l p} j \omega+1}=\frac{k_{c l}\left(1-j T_{l p} \omega\right)}{\left(T_{l p} \omega\right)^{2}+1}
$$

Equivalentstiffness and damping of the rotor can be obtained:

$$
\left\{\begin{array}{l}
k=-k_{i} \frac{k_{c l}}{\left(T_{l p} \omega\right)^{2}+1} \\
d=k_{i} \frac{k_{c l} T_{l p}}{\left(T_{l p} \omega\right)^{2}+1}
\end{array}\right.
$$

The dynamic stiffness of the rotor can be obtained:

$$
K_{d y}=\frac{\sqrt{\left(m T_{l p}{ }^{2} \omega^{4}+m \omega^{2}+k_{i} k_{c l}\right)^{2}+\left(k_{i} k_{c l} T_{l p} \omega\right)^{2}}}{\left(T_{l p} \omega\right)^{2}+1}
$$
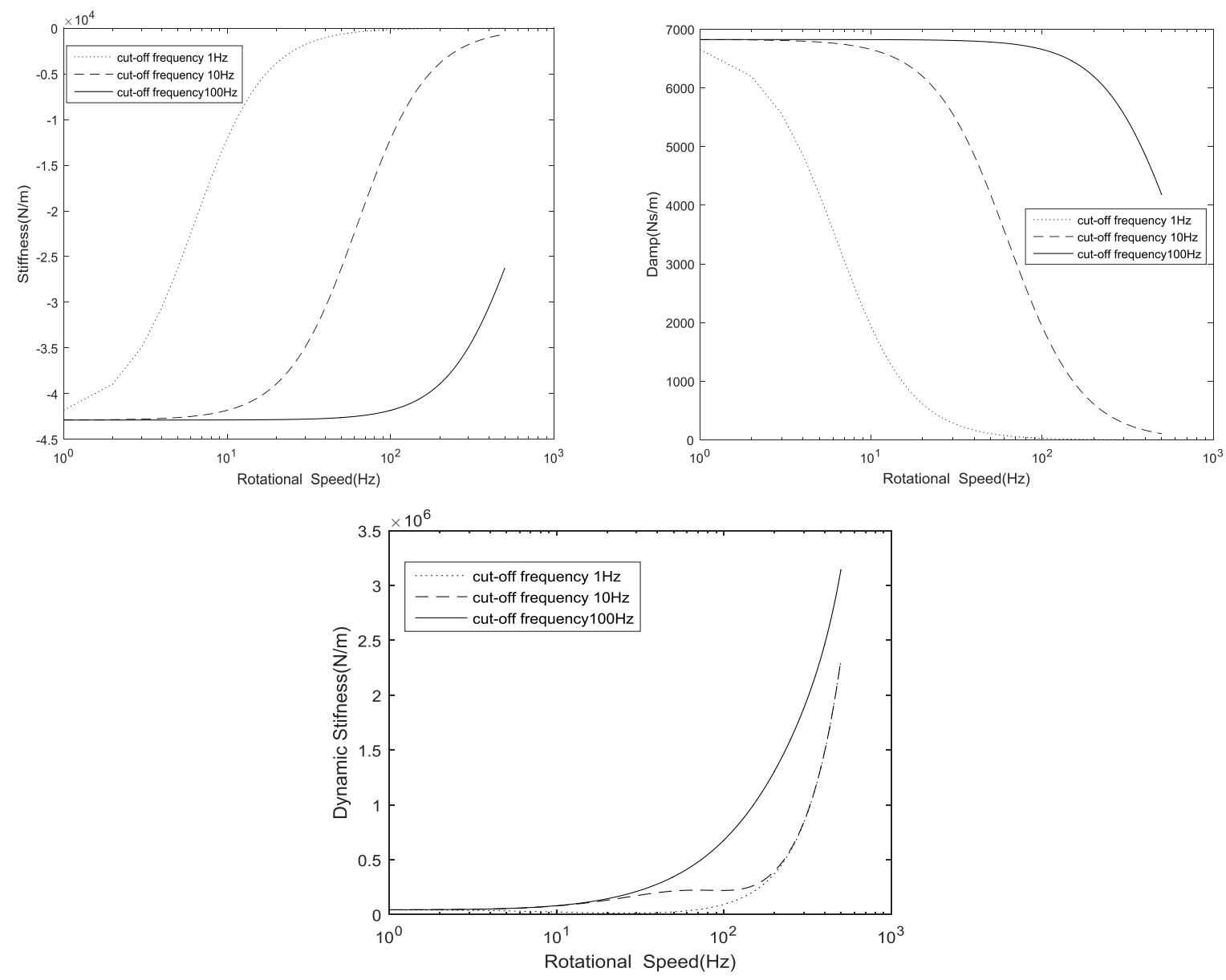

Figure 5.Equivalent stiffness, equivalent damping and dynamic stiffness of the rotor with different cut-off frequency 
As can be seen from Figure 5, as a result of the positive feedback cross control with low-pass characteristic, the value of the equivalent stiffness and damping approach to zero with the increase of rotational speed. And the cut-off frequency of the transfer function has significant influence on stiffness and damping in the low frequency range.

The dynamic stiffness of the rotor tends to be a positive constant in the low and medium frequency range, and the influence of the cut-off frequency is small. With the increase of the rotational speed, the dynamic stiffness increases continuously. In the high frequency range, a higher cut-off frequency will correspond to a larger value of dynamic stiffness.

\section{Negative High-Pass disPlacement Cross Feedback Path}

For the nutation of the rotor, negative displacement cross feedback with high-pass characteristics, namely $^{G_{h}=\frac{k_{c h} s}{T_{h p} s+1}}$, has low gain in low frequency range, which can damp the nutation effectively while the damping of the precession is small.

$$
G_{h}(j \omega)=\frac{k_{c h} j \omega}{T_{h p} j \omega+1}=\frac{j k_{c h} \omega+k_{c h} T_{h p} \omega^{2}}{\left(T_{h p} \omega\right)^{2}+1}
$$

Equivalentstiffness and damping of rotor can be obtained:

$$
\left\{\begin{array}{l}
k=-k_{i} \frac{k_{c h} T_{h p} \omega^{2}}{\left(T_{h p} \omega\right)^{2}+1} \\
d=-k_{i} \frac{k_{c h}}{\left(T_{h p} \omega\right)^{2}+1}
\end{array}\right.
$$

The dynamic stiffness of the rotor can be obtained:

$$
K_{d y}=\frac{\sqrt{\left(m T_{l p}^{2} \omega^{4}+m \omega^{2}+k_{i} k_{c h} T_{h p} \omega^{2}\right)^{2}+\left(k_{i} k_{c h} \omega\right)^{2}}}{\left(T_{h p} \omega\right)^{2}+1}
$$

As can be seen from Figure 6, as a result of the negative feedback cross control with high-pass characteristic, the equivalent stiffness approaches zero and the equivalent damping tends to be a positive constant in low and medium frequency range. And the cut-off frequency of the transferfunctiononly works in high frequency.

The dynamic stiffness of the rotor tends to be zero in the low and medium frequency range, and the influence of the cut-off frequency is small. With the increase of the rotational speed, the dynamic stiffness increases continuously. In the high frequency range, cut-off frequency has little effect on the value of dynamic stiffness. 

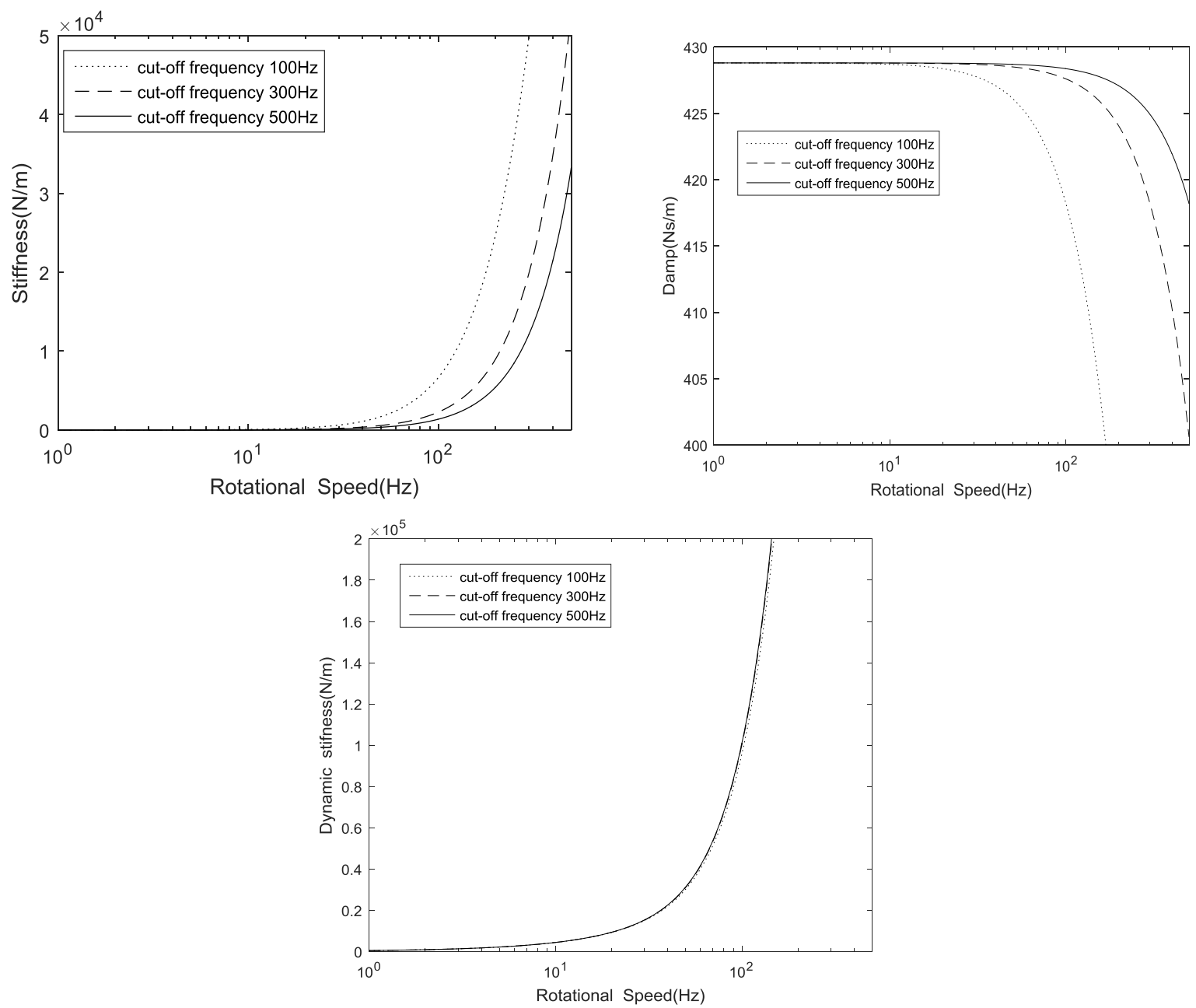

Figure 6.Equivalent stiffness, equivalent damping and dynamic stiffness of the rotor with different cut-off frequency

\section{Combination of Positive Low-Pass and Negative High-Pass Displacement Cross Feedback}

Combining the positive low-pass with negative high-pass displacement cross feedback, the transfer function of crossfeedback path can be written as :

$$
G(\mathrm{~s})=\frac{k_{c l}}{T_{l p} s+1}+\frac{k_{c h} s}{T_{h p} s+1}
$$

Equivalentstiffness and damping of the rotor can be obtained :

$$
\left\{\begin{array}{l}
k=-k_{i} \frac{k_{c l}}{\left(T_{l p} \omega\right)^{2}+1}-k_{i} \frac{k_{c h} T_{h p} \omega^{2}}{\left(T_{h p} \omega\right)^{2}+1} \\
d=k_{i} \frac{k_{c l} T_{l p}}{\left(T_{l p} \omega\right)^{2}+1}-k_{i} \frac{k_{c h}}{\left(T_{h p} \omega\right)^{2}+1}
\end{array}\right.
$$

According to Equation (8), the dynamic stiffness can be obtained. 

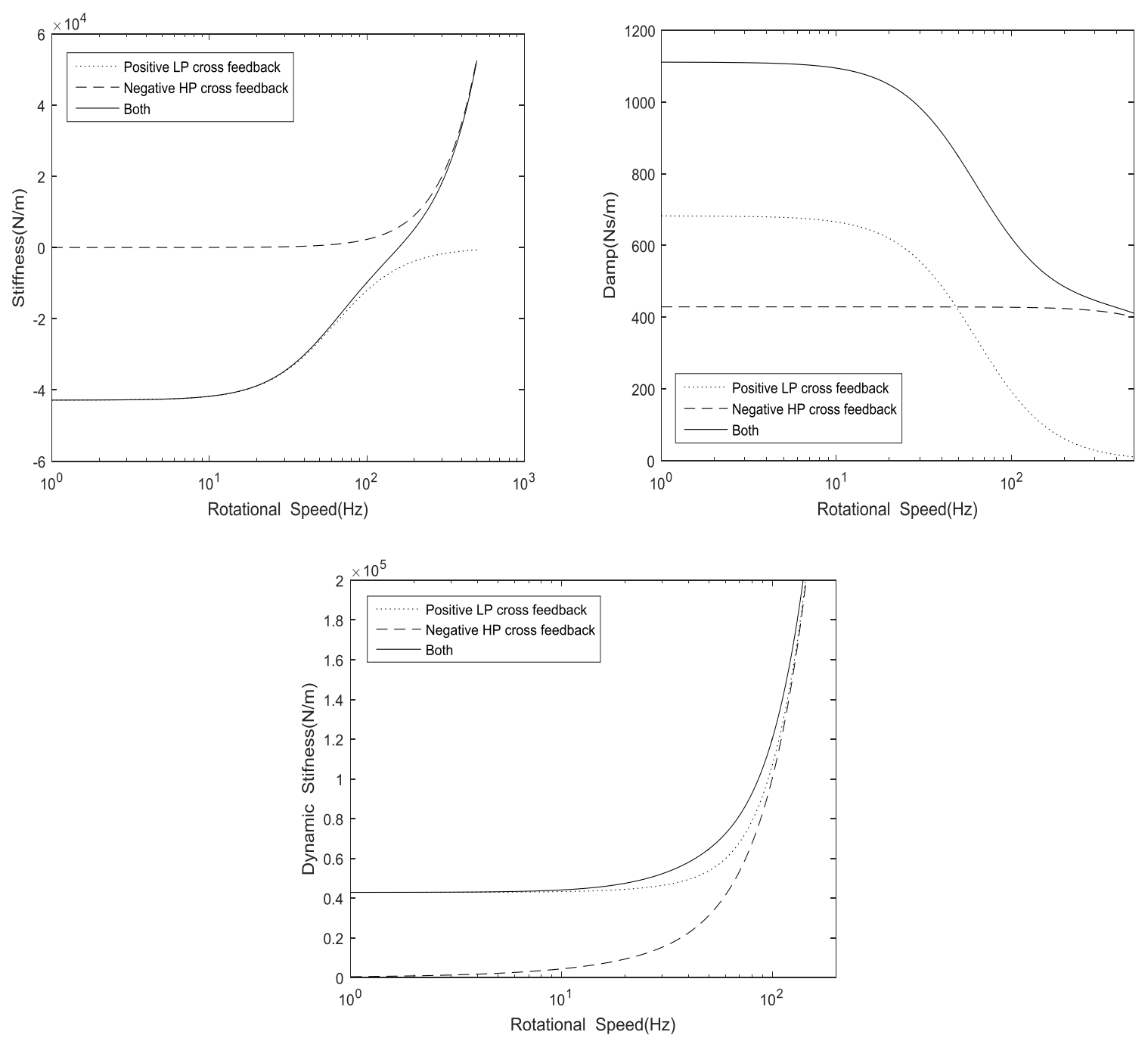

Figure 7. Equivalent stiffness, equivalent damping and dynamic stiffness of the rotor

Figure 7 shows the comparison among the equivalent stiffness, equivalent damping and dynamic stiffness characteristics of the rotor in three different cases. When combining the low-pass positive with the high-pass negative displacement cross feedback, the equivalent stiffness of the rotor is approximately equal to be a negative constant in low frequency. With the increase of rational speed, equivalent stiffness of the rotor approaches zero, and then continues to increase positively; The equivalent damping of the rotor is approximately constant in low and medium frequency range, and decreases with the increase of the rotational speed in the high frequency range; The dynamic stiffness of the rotor is approximately constant in the low and medium frequency range, and increases with the speed increasing.

\section{Conclusion}

The results show that, the cross feedback paths in the controller of the magnetic bearing system, change the stiffness and damping characteristics of magnetic bearing in different frequency range. The rotor dynamic characteristics can be designed for different application with high robustness and dynamic performance.

Supporting characteristics of the rotor can be obtained precisely with the structure of system controller available. Stiffness and damping of each degree of freedom (DOF) can provide convenience for further research on dynamic performance analysis and modal parameter identification test in magneticsuspensionrotorsystem. 


\section{Acknowledgments}

This paper is supported by the National Natural Science Foundation of China (No.51275261) and National S\&T Major Project (No.ZX069). The authors would also like to gratefully acknowledgment referees for their many insightful comments.

\section{References}

1. Bleuler $\mathrm{H}$, Cole $\mathrm{M}, \mathrm{Keogh} \mathrm{P}$, et al. Magnetic bearings: theory, design, and application to rotating machinery [M]. Springer Science \& Business Media, 2009.

2. Ahrens M, Kucera L. Cross feedback control of a magnetic bearing system: Controller design considering gyroscopic effects[J]. 1996.

3. Habermann H, Liard G. An active magnetic bearing system [J]. TRIBOLOGY international, 1980, 13(2): 85 .

4. Humphris R R, Kelm R D, Lewis D W, et al. Effect of control algorithms on magnetic journal bearing properties[J]. Journal of Engineering for Gas Turbines and Power, 1986, 108(4): 624-632.

5. Xiping $\mathrm{W}$, Lijing $\mathrm{Z}$, Liang $\mathrm{Y}$, et al. Investigation on dynamic performance of active magnetic bearing rotor system [J]. Journal of Mechanical Engineering, 2001, 37(11): 7-12.

6. Zhao J, Zhou Y, Shi Z, et al. Automatic measurement of stiffness and damping of active magnetic bearings[J]. Journal of Mechanical Engineering, 2010, 46(20): 48-52.

7. Bauomy H S. Stability analysis of a rotor-AMB system with time varying stiffness[J]. Journal of the Franklin Institute, 2012, 349(5): 1871-1890.

8. Sayyad F B, Gadhave N D. Variable stiffness type magnetic vibration absorber to control the vibration of beam structure [J]. Journal of Vibration and Control, 2014, 20(13): 1960-1966.

9. Zheng S, Han B, Wang Y, et al. Optimization of damping compensation for a flexible rotor system with active magnetic bearing considering gyroscopic effect [J]. IEEE/ASME Transactions on Mechatronics, 2015, 20(3): 1130-1137.

10. $\mathrm{Xu} \mathrm{Y,} \mathrm{Zhou} \mathrm{J,} \mathrm{Di} \mathrm{L,} \mathrm{et} \mathrm{al.} \mathrm{Active} \mathrm{magnetic} \mathrm{bearings} \mathrm{dynamic} \mathrm{parameters} \mathrm{identification} \mathrm{from}$ experimental rotor unbalance response [J]. Mechanical Systems and Signal Processing, 2017, 83: 228-240. 